\title{
Sequential Treatment with Eldecalcitol After PTH Improves Bone Mechanical Properties of Lumbar Spine and Femur in Aged Ovariectomized Rats
}

\author{
Sadaoki Sakai ${ }^{1,2} \cdot$ Hiromi Hongo $^{3} \cdot$ Tomomaya Yamamoto $^{4} \cdot$ Tomoka Hasegawa $^{3} \cdot$ Satoshi Takeda $^{1} \cdot$ Hitoshi Saito $^{2}$. \\ Koichi Endo ${ }^{5} \cdot$ Kenji Yogo $^{1} \cdot$ Norio Amizuka $^{3}$
}

Received: 30 August 2018 / Accepted: 17 November 2018 / Published online: 22 November 2018

(c) The Author(s) 2018

\begin{abstract}
Parathyroid hormone (PTH) analogs have a powerful anabolic effect on bone and are used in the treatment of patients with severe osteoporosis. However, there are limitations to how long they can be safely administered. Withdrawal of PTH results in the cancelation of its effects, necessitating subsequent treatment to maintain the bone quantity and quality. This study assessed the effects of Eldecalcitol (ELD), an active vitamin D3 derivative, after PTH in estrogen-deficient osteoporotic rats. Six-month-old female rats were ovariectomized, and PTH administration was started 7 weeks later. After 4 weeks of PTH treatment, the animals were divided into three groups and either continued to receive PTH (PTH-PTH), or were switched to ELD (PTH-ELD) or vehicle (PTH-Veh) for an additional 4 weeks. In the femur, increased BMD by 4 weeks treatment of PTH was significantly reduced in PTH-Veh but not in PTH-PTH and PTH-ELD. The same tendency was observed in the lumbar vertebrae. MicroCT imaging and histomorphometry analysis revealed that the favorable bone structure changes by PTH administration were also maintained in the femurs and tibias of the PTH-PTH and PTH-ELD groups. Increased bone strength by 4-week treatment of PTH in lumber also maintained in PTH-ELD. Furthermore, minimodeling was observed in the PTH-ELD group. These results demonstrate that treatment with ELD sequentially following PTH prevented the bone quantity and strength reduction that accompanies PTH withdrawal in estrogen-deficient rats.
\end{abstract}

Keywords Eldecalcitol $\cdot$ Minimodeling $\cdot$ Osteoporosis $\cdot$ Ovariectomized rat $\cdot$ Parathyroid hormone

\section{Introduction}

Osteoporosis is a skeletal disorder characterized by a reduction in bone strength and an increase in bone fracture risk. The goal of osteoporosis treatment is to reduce bone fracture risk by preventing loss of bone quantity or quality. Drugs for the treatment of osteoporosis fall into two major classes, anti-bone-resorptive agents and anabolic agents. There are several types of anti-resorptive treatments such as selective estrogen receptor modulators (SERMs), bisphosphonates, and active vitamin D analogs. In contrast, the parathyroid hormone (PTH) analogs PTH1-34 and PTH1-84 are currently the only approved agents categorized as anabolic agents [1].

PTH stimulates bone formation by increasing osteoblast numbers via osteoblastogenesis [2], thereby increasing the bone mineral density (BMD) of the spine and reducing the risk of vertebral fractures in postmenopausal women with prevalent vertebral fractures [3]. Favorable effects of PTH on 
BMD and fracture risk are reported even in male osteoporosis patients [4]. Because PTH is highly potent in increasing $\mathrm{BMD}$ and reducing fracture risk, it is used for the treatment of severe osteoporosis in patients with high fracture risk.

However, it is reported that long-term usage of PTH induces osteosarcoma in rats [5]. Although the risk of carcinogenicity in humans is not clear, the clinical administration of PTH is limited to no more than 2 years, and unfortunately the increase in lumbar spinal BMD elicited by PTH treatment is not maintained after PTH withdrawal $[6,7]$. Therefore, sequential treatments for osteoporosis are required after discontinuation of PTH treatment [8-10]. Several reports have demonstrated the usefulness of sequential treatment with anti-bone-resorptive agents such as SERMs or bisphosphonate after PTH treatment in animal models [11, 12]. We also reported that ibandronate, a nitrogen-containing bisphosphonate, suppressed bone turnover and maintained BMD in ovariectomized (OVX) rats that had been pretreated with PTH [13].

PTH enhances bone formation by two different mechanisms, remodeling (bone formation associated with bone resorption) and minimodeling (bone formation independent of bone resorption), and which mechanism is adopted depends on the frequency of PTH administration [14]. Eldecalcitol (ELD) is an analog of active vitamin $\mathrm{D}_{3}$ used for osteoporosis treatment in Japan [15] and is now under development in China. ELD is reported to enhance minimodelingbased bone formation and inhibit bone resorption [16, 17] and to enhance bone maturation processes such as mineralization and enzymatic crosslinking of collagen [18]. Because of these effects on the enhancement of bone formation and maturation and on the inhibition of bone resorption, treatment with ELD after PTH withdrawal seems to be a reasonable option for osteoporosis treatment. However, there are no reported studies on the influence of sequential treatment with active vitamin $\mathrm{D}_{3}$ after PTH treatment.

In the current study, we examined the effects of sequential treatment with ELD following PTH in estrogen-deficient OVX rats.

\section{Materials and Methods}

\section{Animal Experiments}

Female Wistar-Imamichi rats (6 months old) were obtained from the Institute for Animal Reproduction (Ibaraki, Japan). Totally 76 animals were used in this study. Animals were given free access to tap water and standard rodent chow (CE2; CLEA Japan Inc., Tokyo, Japan) for the duration of the experiment. After acclimatization for 1 or 2 weeks to recover from shipping-related stress, rats were ovariectomized (OVX group) or underwent a sham operation (Sham group). Seven weeks later (at time point 'Pre'), treatment with 1-34 PTH (PTH) (Peptide Institute, Inc., Osaka, Japan) was initiated. PTH dissolved in citrate-phosphate buffer was subcutaneously administered at a dose of $10 \mu \mathrm{g} / \mathrm{kg} / \mathrm{day}$, five times a week. After 4 weeks of treatment with PTH (PTH group), at time point ' $W \mathrm{~K}$ 4', animals were divided into three groups. One group continued to receive the same dose of PTH (PTH-PTH group) and the other two groups were administered eldecalcitol (PTH-ELD group) or vehicle (PTH-Veh group) for an additional 4 weeks (until time point 'Wk 8'). ELD dissolved in medium-chain fatty acid was administered orally at a dose of $20 \mathrm{ng} / \mathrm{kg}$, five times a week. The doses of PTH and ELD were determined by previous reports as a suitable dose to evaluate the bone volume or strength in OVX rats $[13,19]$. Animals were euthanized by exsanguination under isoflurane anesthesia and their lower limbs and lumbar spines were collected at time points of Pre (Sham-Pre group; $\mathrm{N}=7$, OVX-Pre group; $\mathrm{N}=8$ ), Wk 4 (Sham; $\mathrm{N}=8$, OVX; $\mathrm{N}=10$, and $\mathrm{PTH} ; \mathrm{N}=10$ groups), and Wk 8 (PTH-PTH; $\mathrm{N}=11$, $\mathrm{PTH}-\mathrm{ELD} ; \mathrm{N}=11$, and $\mathrm{PTH}-\mathrm{Veh} ; \mathrm{N}=11$ groups). Tetracycline (oxytetracycline hydrochloride, $20 \mathrm{mg} / \mathrm{kg}$ ) and calcein $(6 \mathrm{mg} / \mathrm{kg})$ were injected subcutaneously for bone labeling at 6 days and 2 days, respectively, prior to rats being euthanized. Every one animal in Wk 8 groups was made perfusion fixation by $4 \%$ paraformaldehyde in $0.1 \mathrm{M}$ cacodylate buffer for staining of alkaline phosphatase and tartrate-resistant acid phosphatase described following and removed from evaluation of other bone parameters.

\section{Dual-Energy X-ray Absorptiometry}

BMD of the second to fourth lumbar vertebrae (L2-L4) and right femur was determined by dual-energy X-ray absorptiometry (DCS-600 EX; Hitachi, Tokyo, Japan). After dualenergy X-ray images were obtained, the long axis of the femur was divided into ten parts (numbered F1-F10 from the proximal to distal ends) and the BMD of the whole femur (F1-F10), proximal femur (F1-F3), middle femur (F4-F7), and distal femur (F8-F10) was calculated.

\section{Micro X-ray CT Imaging}

Micro X-ray computed tomography (microCT) was carried out using the R_mCT2 system (Rigaku Co., Tokyo, Japan) using $90 \mathrm{kV}$ X-ray tube voltage, $160 \mu \mathrm{A} \mathrm{X}$-ray tube current with $0.06 \mathrm{~mm}$ cupper and $0.5 \mathrm{~mm}$ aluminum filter, and structural parameters were analyzed with TRI/3D-BON (Ratoc System Engineering Co. Ltd., Tokyo, Japan). Horizontal sections of the distal femur (2500-3000 $\mu \mathrm{m}$ from the growth plate) with a voxel size of $20 \mu \mathrm{m}$ and 3 min scanning time were used for trabecular and cortical bone analysis. The analyzed parameters were trabecular volumetric BMD (BMD, $\mathrm{mg} / \mathrm{cm}^{3}$ ), trabecular bone volume/tissue volume (BV/TV, 
$\%)$, trabecular thickness (Tb.Th, $\mu \mathrm{m})$, trabecular number (Tb.N, /mm), total strut length/tissue volume (TSL/TV, 1/ $\mathrm{mm}^{2}$ ) and trabecular separation (Tb.Sp, $\mu \mathrm{m}$ ).

\section{Bone Strength Measurement}

The strength of trabecular and cortical bone was assessed by means of a compression test for the L5 lumbar vertebra and a 3-point bending test for the middle femur. The vertebral arch and disk were removed from each L5 lumbar vertebra and vertebral bodies were trimmed to a length of $5 \mathrm{~mm}$ on the vertical axis. The trimmed vertebral bodies were placed in a bone strength tester (TK-252C; Muromachi Kikai Co., Ltd., Tokyo, Japan), and compressive strength was measured by pressure at a speed of $2.5 \mathrm{~mm} / \mathrm{min}$. The 3 -point bending strength of the femur was measured by the same tester. The midpoint of the left femur was placed on a holding device, and the supports were located $12 \mathrm{~mm}$ apart. The bending force was calculated at a speed of $20 \mathrm{~mm} / \mathrm{min}$ until fractures occurred. From the load-displacement curve, the ultimate load $(\mathrm{N})$, stiffness $(\mathrm{N} / \mathrm{mm})$, and energy $(\mathrm{mJ})$ were obtained.

\section{Bone Histomorphometry Analysis}

Bone histomorphometry analysis was performed as previously reported [20]. Briefly, the left tibia was fixed in $70 \%$ ethanol and stained according to the method of Villanueva [21]. After dehydration with ethanol and acetone, the samples were embedded in methyl methacrylate (Wako Pure Chemical Industries Ltd., Osaka, Japan). Frontal plane sections of the proximal tibia, $5 \mu \mathrm{m}$ thick, were obtained with a microtome (Supercut 2050; Reichert-Jung, Heidelberg, Germany). Images obtained under optical and fluorescence microscopes were recorded with a digital camera, and the primary histomorphometric parameters were measured with an image analyzing system (Cosmozone 1SA; Nikon Co., Tokyo, Japan). Nomenclature, symbols, and units used in this study are those described in the Report of the American Society for Bone and Mineral Research Nomenclature Committee [22].

\section{Staining for Tissue-Nonspecific Alkaline Phosphatase (ALP) and Tartrate-Resistant Acid Phosphatase (TRAP)}

The samples of tibia were decalcified in a $10 \%$ ethylenediaminetetraacetic acid disodium salt (EDTA-2Na) solution. Decalcified samples were then dehydrated in ascending alcohol solutions before embedding in paraffin and sectioning. After inhibition of endogenous peroxidase activity with methanol containing $0.3 \%$ hydrogen peroxidase for $30 \mathrm{~min}$, deparaffinized sections were examined for tissue-nonspecific alkaline phosphatase (ALP) as reported previously [23]. In brief, after the inhibition of endogenous peroxidase activity, sections were incubated with rabbit polyclonal antisera against ALP [24], and connected ALP antibodies were captured by horseradish peroxide-conjugated anti-rabbit $\operatorname{IgG}$ (DakoCytomation, Glostrup, Denmark) and visualized by diaminobenzidine tetrahydrochloride (DAB). Tartrate-resistant acid phosphatase (TRAP) activity was also detected as previously reported [25]. After rinsing with PBS, decalcified sections were incubated in a mixture of naphthol AS-BI phosphate (Sigma-Aldrich Japan, Tokyo, Japan), Fast Red Violet LB salt (Sigma-Aldrich), and $100 \mathrm{mM}_{\mathrm{L}}$-tartaric acid diluted in $0.1 \mathrm{M}$ sodium acetate buffer ( $\mathrm{pH}$ 5.0).

Decalcified tibias were post-fixed with $1 \%$ osmium tetraoxide with a $0.1 \mathrm{M}$ cacodylate buffer for $4 \mathrm{~h}$ at $4{ }^{\circ} \mathrm{C}$, dehydrated in ascending acetone solutions, and embedded in epoxy resin (Epon 812; Taab, Berkshire, UK). Semi-thin sections were prepared with an ultramicrotome, and then stained with toluidine blue for light microscopy.

\section{Statistical Analysis}

All values are shown as means and standard deviation (SD). Statistical analysis was performed with the JMP statistical analysis software package (SAS Institute Japan Ltd., Tokyo, Japan). The significance of differences was determined by using unpaired $t$-test (Wk 4 OVX vs. Wk 4 PTH) or Dunnett's multiple comparison test (Wk 4 PTH vs. Wk 8 PTH-PTH, PTH-ELD, and PTH-Veh). $p$-values $<0.05$ were considered significant.

\section{Results}

\section{Bone Mineral Density Assessment of Lumbar Spine and Femur by Dual-Energy X-ray Absorptiometry}

BMD of the lumbar spine (L2-L4) and femur at time point 'Pre' (7 weeks after ovariectomy or sham operation), at Wk 4 (after 4 weeks of PTH treatment from point 'Pre'), and at Wk 8 (after an additional 4 weeks from Wk 4 with either PTH continued, PTH switched to ELD, or PTH withdrawn) were measured using dual-energy X-ray absorptiometry (Fig. 1). Four-week administration of PTH after 7 weeks of estrogen deficiency increased BMD (measured at Wk 4) in both the lumbar spine (Fig. 1a) and the whole, proximal and distal femur (Fig. 1b, c, e) compared with the vehicle-treated OVX group. In the PTH-Veh group (4-week withdrawal of PTH after 4 weeks of PTH administration), BMD measured at Wk 8 was reduced in the whole femur and distal femur and tended to be reduced in all other regions compared with 4-week treatment with PTH measured at Wk 4. In the PTH-PTH group, which received continuous treatment with PTH, femur BMD increased after 4 weeks of additional PTH 
A L2-L4 BMD (mg/cm²)

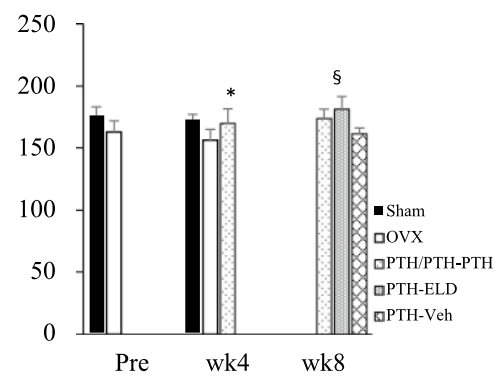

B Whole Femur BMD $\left(\mathrm{mg} / \mathrm{cm}^{2}\right)$

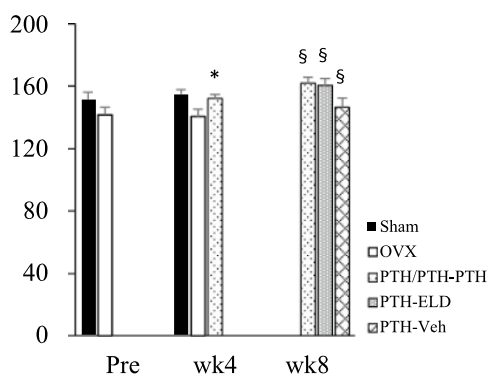

C Proximal Femur BMD $\left(\mathrm{mg} / \mathrm{cm}^{2}\right)$

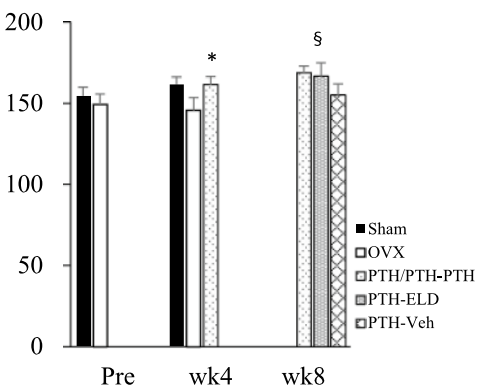

D Middle Femur BMD $\left(\mathrm{mg} / \mathrm{cm}^{2}\right)$

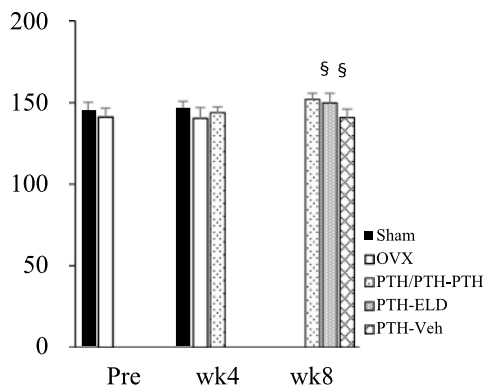

E Distal Femur BMD $\left(\mathrm{mg} / \mathrm{cm}^{2}\right)$

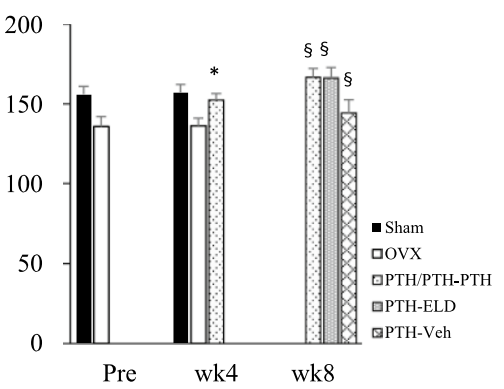

Fig. 1 Bone mineral density of lumbar spine (L2-L4) and femur in rats were measured by dual-energy X-ray absorptiometry. Each femur was divided into ten parts (numbered F1-F10 from the proximal to distal ends), then BMD of the whole femur (F1-F10, b), proximal femur (F1-F3, c), middle femur (F4-F7, d), and distal femur (F8F10, e) was calculated. Data are presented as mean $+\mathrm{SD}(N=7$ in pre of Sham, $N=8$ in pre of OVX and Wk 4 of Sham, and $N=10$ in other groups in A. $N=9$ in Wk 4 of PTH in b-e because one sample was missing, No. of other groups in $\mathbf{b}-\mathbf{e}$ are same as $\mathbf{a}) .{ }^{*}$ Significant difference versus vehicle-treated OVX rats at Wk4 (unpaired $t$-test, $p<0.05$ ). ${ }^{\S}$ Significant difference versus PTH group at Wk 4 (Dunnett's multiple comparison test, $p<0.05$ ) treatment compared with the PTH group at Wk 4. Switching to ELD after 4 weeks of PTH treatment (the PTH-ELD group) increased lumbar and femoral BMD (except for in the proximal femur).

\section{Histological Observation of Metaphyseal Trabecular Bone in Proximal Tibia}

We made several qualitative evaluation using tissue sample staining by Images of hematoxylin and eosin (HE) stained sections of the proximal metaphyseal tibia are shown in Fig. 2. Estrogen deficiency for 7 weeks (Fig. 2b) showed low level of in trabecular bone, and an additional 4 weeks of estrogen deficiency (Fig. 2d) resulted in markedly decreased bone mass (Fig. 2c). Four weeks of treatment with PTH (Fig. 2e) seemed to rescue the trabecular bone that had been reduced by estrogen deficiency. At Wk 8, only a little trabecular bone can be seen in rats from the PTH-Veh group (Fig. 2F), while abundant thick trabecular bone can be observed in rats from the PTH-PTH group (Fig. 2g). Many trabecular bone in rats from the PTH-ELD group (Fig. 2h) was also large (Fig. 2f). Thick cortical bone was observed in both the PTH-PTH group (Fig. 2g) and the PTH-ELD group (Fig. 2h).

TRAP-positive cells, which are considered to be osteoclasts, were mainly located under the growth plate in all groups, with few osteoclasts in secondary trabecular bone (Fig. 3a-c). However, osteoclasts were abundant in the PTH-Veh group (Fig. 3d) and PTH-PTH group (Fig. 3e) and rare in the PTH-ELD group (Fig. 3f). ALP-positive cells, which are considered to be osteoblasts were also mainly located under the growth plate (Fig. 3g-i). In PTH-PTH group, ALP-positive cells were observed even on the trabecular bone surface and it made two layers (Fig. 3k). ALPpositive cells were observed even on the trabecular bone surface of PTH-ELD group (Fig. 31), whereas it were not observed in the sample from PTH-Veh group (Fig. 3j).

\section{Trabecular Bone Structure Analysis by microCT}

The structure of trabecular bone in the distal femur was analyzed by using microCT. Tissue BMD (bone mineral content per bone volume) in the PTH group at Wk 4 was significantly higher than in the vehicle-treated OVX group 


\section{HE staining}

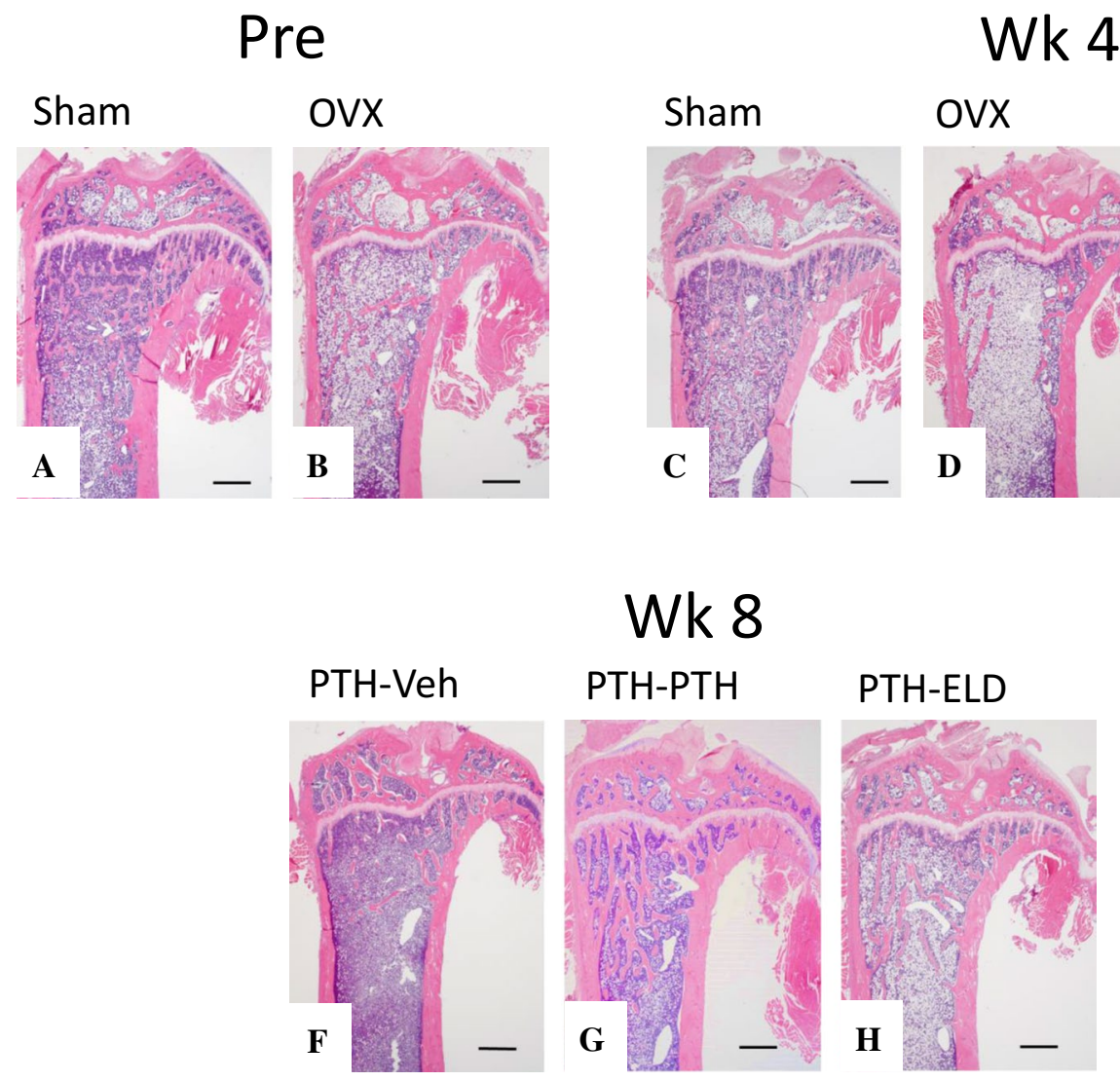

Fig. 2 Typical images of HE staining of proximal tibias in each group. Scale bars show $50 \mu \mathrm{m}$

(Fig. 4a). Continuous treatment with PTH over an additional 4 weeks (PTH-PTH group at Wk 8) significantly increased tissue BMD of trabecular bone compared with that of the $\mathrm{PTH}$ group at Wk 4. Tissue BMD in the PTH-Veh group at Wk 8 was significantly lower than that in the PTH group at Wk 4. Tissue BMD in the PTH-ELD group at Wk 8 remained at the level in the PTH group at Wk 4. BV/TV (bone volume/tissue volume) also showed similar changes, but the changes with each treatment at Wk 8 (vs. the PTH group at Wk 4) did not reach a significant level (Fig. 4b). Tb.Th (trabecular thickness) was strongly increased by PTH treatment in a treatment-period-dependent manner, but decreased after withdrawal of PTH (Fig. 4c). Tb.Th in PTH-ELD was not show significant difference with in the PTH group at Wk 4. PTH treatment also increased Tb.N (trabecular number) and TSL/TV (total strut length/tissue volume) but there were no significant differences between 4-week administration and 8-week administration (PTH group vs. PTH-PTH group) (Fig. 4d, e). The values of Tb.N and TSL/TV in the PTH-ELD group were not different significantly from in the PTH-PTH group. In contrast, $\mathrm{Tb} . \mathrm{Sp}$ (trabecular separation) was increased by OVX but there was no statistically significant difference between any of the groups treated with anti-osteoporotic agents (Fig. 4f).

\section{Bone Histomorphometry of the Proximal Tibia}

Data on bone histomorphometry of the proximal tibia are shown in Table 1. BV/TV in the PTH-treated group was increased at $\mathrm{Wk} 4$, whereas it was reduced by 4-week withdrawal (PTH-Veh group at Wk 8) compared with BV/ $\mathrm{TV}$ in the PTH group at Wk 4. BV/TV in the PTH-ELD group at Wk 8 was not show significant difference from that in PTH group at Wk 4. Tb.Th showed the same tendency as that of BV/TV. PTH treatment reduced Tb.Sp at Wk 4 (PTH group) and this reduced Tb.Sp was kept until Wk 8 (PTH-PTH group). The reduction of Tb.Sp by PTH was maintained in the PTH-ELD group, but it seemed to be partially canceled by the cessation of PTH treatment. Although BS/BV (bone surface/bone volume) was significantly decreased by PTH at Wk 4, it was increased by PTH withdrawal. Switching to ELD kept BS/BV as low as it was after PTH treatment at Wk 4. Bone formation parameters such as osteoblast surface/bone surface (Ob.S/ 


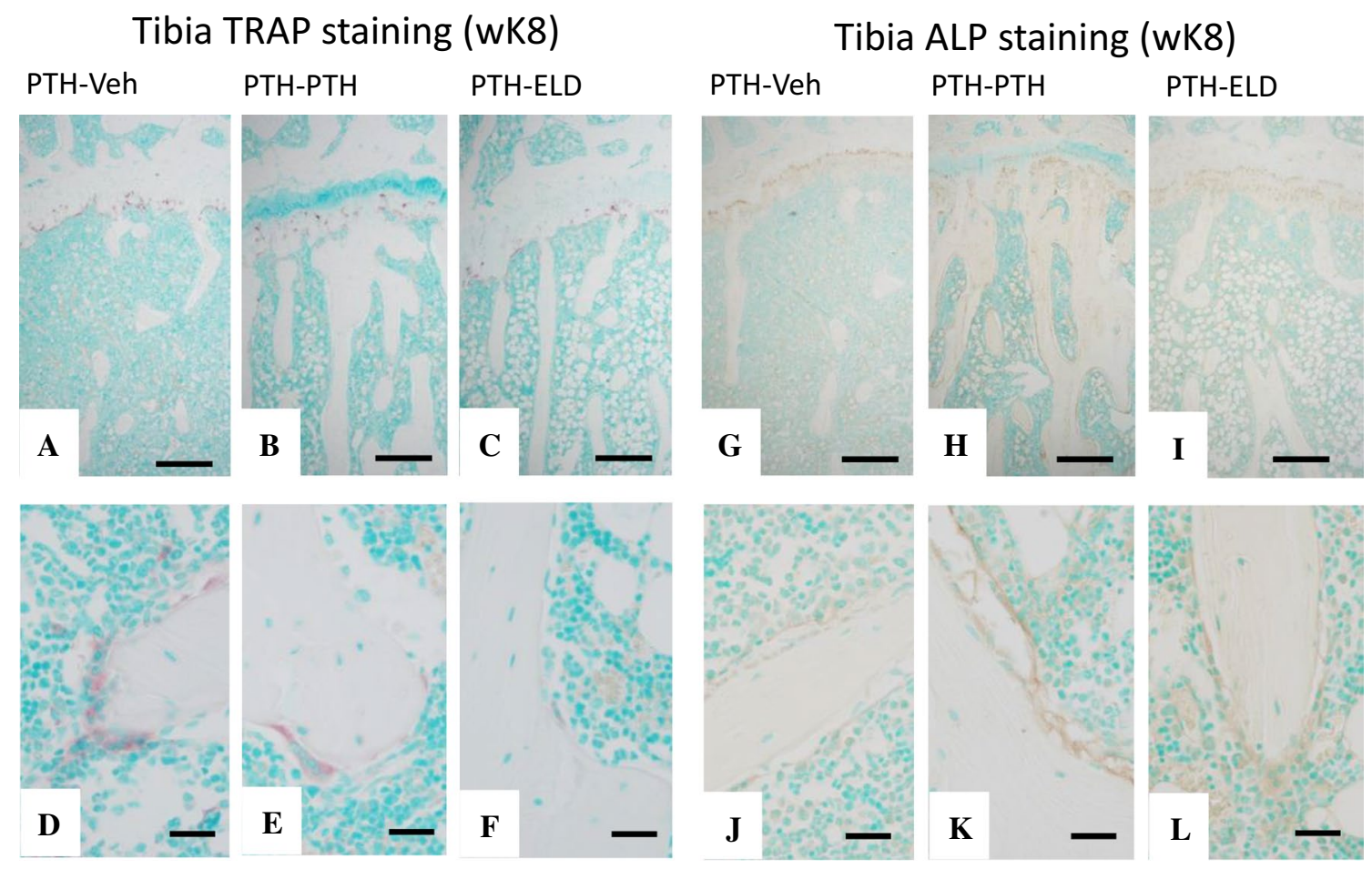

Fig. 3 Typical images of TRAP staining (a-f) and ALP staining ( $\mathbf{g}-\mathbf{l})$ on proximal tibias at Wk 8 in each group. Scale bars show $100 \mu \mathrm{m}$ in upper line $(\mathbf{a}-\mathbf{c}, \mathbf{g}-\mathbf{i})$ and $20 \mu \mathrm{m}$ in lower line $(\mathbf{d}-\mathbf{f}, \mathbf{j}-\mathbf{l})$

BS), mineralizing surface/bone surface (MS/BS), bone formation rate/bone surface (BFR/BS), and activation frequency (Ac.f) were each increased by 4 weeks of PTH treatment compared with the vehicle-treated OVX group and these parameters after additional 4 weeks of PTH treatment (PTH-PTH group at Wk 8) did not differ from those in PTH at Wk 4 significantly. On the other hand, these bone formation parameters at Wk 8 were reduced in the PTH-ELD group compared with in the PTH group at Wk 4. Withdrawal of PTH increased levels of bone resorption parameters ES/BS (eroded surface/bone surface) and N.Oc/BS (osteoclast number/bone surface).

Fluorescence microscopic observation revealed minimodeling-which is locally convex bone formation (Fig. 5a, b, arrow) as distinguished from remodeling which is bone formation after bone resorption (Fig. 5a arrow head) - in tibial trabecular bone of the PTH-ELD group. In semi-thin sections with toluidine blue staining from the PTH-PTH group (Fig. 5c), cuboidal osteoblasts and abundant pre-osteoblasts were observed on the surface of trabecular bone. In the image of the semithin section from the PTH-ELD group, locally convex bone formation on a smooth cement line without a thick pre-osteoblast layer (i.e., minimodeling) was observed (Fig. 5d, arrow).

\section{Bone Mechanical Property Assessment of Lumbar Vertebra and Femoral Cortical Bone}

Bone mechanical properties were assessed by compression test for the L5 lumbar vertebra and 3-point bending test for the middle femur. With the L5 lumbar vertebra, PTH treatment for 4 weeks improved bone mechanical parameters compared with vehicle treatment. Although the bone mechanical parameters tended to deteriorate with the cessation of PTH (PTH-Veh), switching to ELD (PTH-ELD) maintained or even improved those parameters (Fig. 6a-c). With the left femur, no significant improvement in bone mechanical parameters was observed with PTH treatment, and the results even showed a reduction in stiffness at $\mathrm{Wk} 4$. This reduction in stiffness recovered following PTH cessation. An additional 4 weeks of treatment with ELD (PTH-ELD group) increased ultimate load and stiffness compared with these parameters in the PTH group at Wk 4 (Fig. 6d-f).

\section{Discussion}

PTH analogs are currently the only bone anabolic agents so far approved for treatment of osteoporosis. PTH strongly increases BMD and reduces the risk of bone fracture. 
A Tissue $\mathrm{BMD}\left(\mathrm{mg} / \mathrm{cm}^{3}\right)$

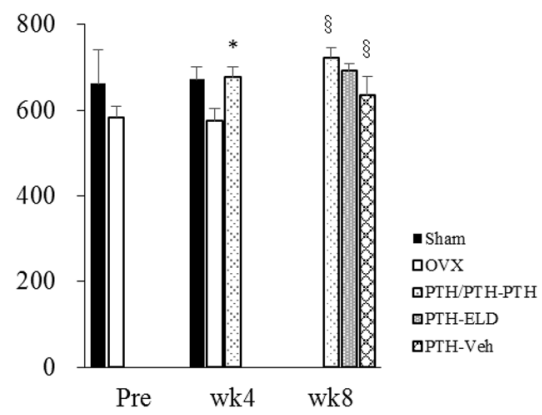

D $\operatorname{Tb} . \mathrm{N}(1 / \mathrm{mm})$

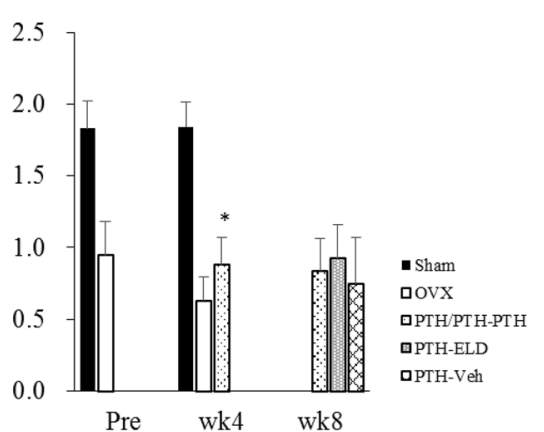

B $\mathrm{BV} / \mathrm{TV}(\%)$

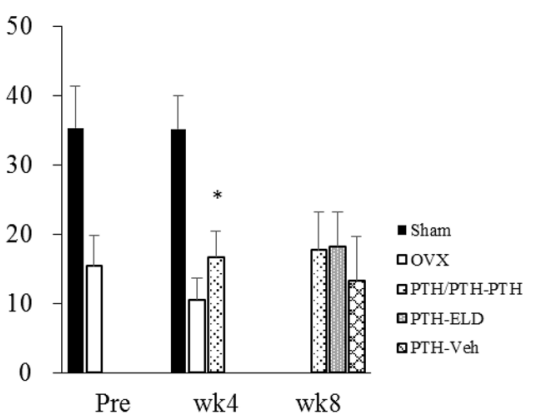

E $\operatorname{TSL} / \mathrm{TV}\left(1 / \mathrm{mm}^{2}\right)$

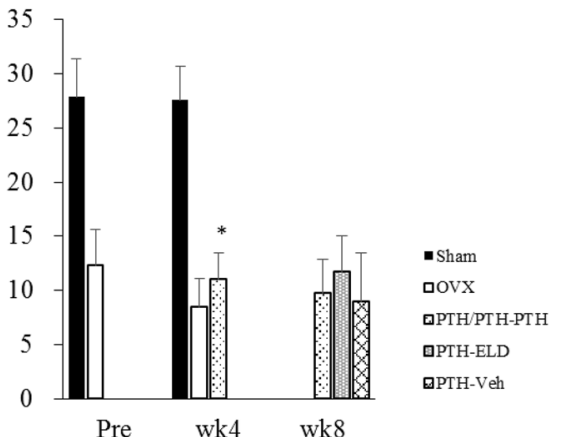

C $\quad \operatorname{Tb} . T h(\mu \mathrm{m})$

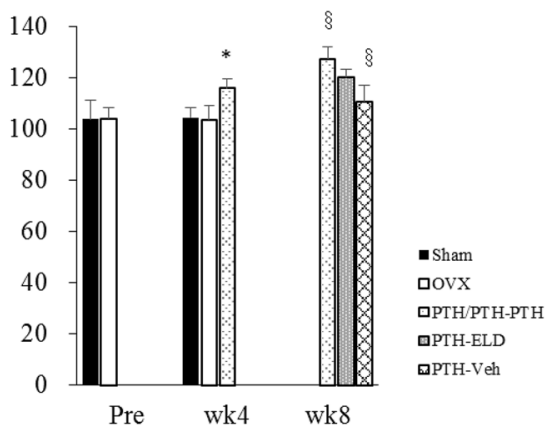

F Tb.Sp $(\mu \mathrm{m})$

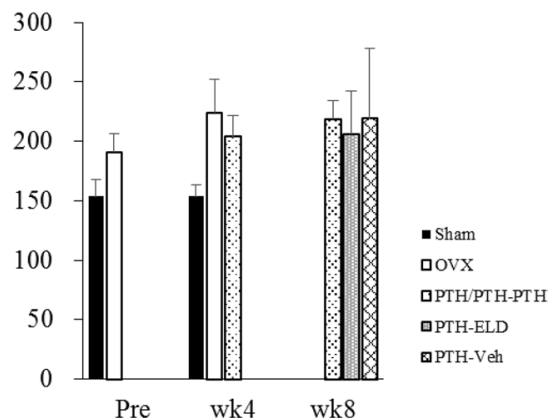

Fig. 4 Structure of trabecular bone in the distal femur was analyzed by using microCT. Data are presented as mean $+\mathrm{SD}(N=7$ in pre of Sham, $N=8$ in pre of OVX and Wk 4 of Sham, and $N=10$ in other groups). *Significant difference vs. vehicle-treated OVX at Wk 4 (unpaired $t$-test, $p<0.05$ ). ${ }^{\S}$ Significant difference versus PTH group at Wk 4 (Dunnett's multiple comparison test, $p<0.05$ ). BV/TV bone volume/tissue volume, Tb.Th trabecular thickness, $T b . N$ trabecular number, $T S L / T V$ total strut length/tissue volume, $T b . S p$ trabecular separation

Table 1 Bone histomorphometry of the proximal tibia

\begin{tabular}{|c|c|c|c|c|c|c|c|c|}
\hline & \multicolumn{2}{|l|}{ Pre } & \multicolumn{3}{|l|}{ Wk 4} & \multicolumn{3}{|l|}{ Wk 8} \\
\hline & Sham & OVX & Sham & Vehicle & PTH & PTH-PTH & PTH-ELD & PTH-Vehicle \\
\hline $\mathrm{BV} / \mathrm{TV}(\%)$ & $29.9 \pm 4.1$ & $20.3 \pm 3.3$ & $33.3 \pm 6.0$ & $18.5 \pm 4.8$ & $24.5 \pm 4.2^{*}$ & $27.8 \pm 6.1$ & $26.5 \pm 3.3$ & $17.1 \pm 3.3^{\S}$ \\
\hline Tb.Th $(\mu \mathrm{m})$ & $73.5 \pm 7.2$ & $64.9 \pm 5.1$ & $78.7 \pm 11.1$ & $70.5 \pm 11.2$ & $84.7 \pm 9.7 *$ & $97.1 \pm 9.2^{\S}$ & $87.3 \pm 6.7$ & $66.4 \pm 7.7^{\S}$ \\
\hline Tb.Sp $(\mu \mathrm{m})$ & $174 \pm 21$ & $259 \pm 31$ & $159 \pm 25$ & $320 \pm 60$ & $267 \pm 52 *$ & $265 \pm 77$ & $245 \pm 31$ & $331 \pm 66$ \\
\hline $\mathrm{BS} / \mathrm{BV}\left(\mu \mathrm{m} / \mu \mathrm{m}^{2}\right) \times 1000$ & $27.4 \pm 2.5$ & $31.0 \pm 2.5$ & $25.9 \pm 3.7$ & $29.0 \pm 4.6$ & $23.9 \pm 2.8$ & $20.8 \pm 2.0^{\S}$ & $23.0 \pm 1.7$ & $30.5 \pm 3.5^{\S}$ \\
\hline OS/BS (\%) & $16.5 \pm 4.8$ & $31.1 \pm 4.8$ & $5.4 \pm 3.4$ & $30.3 \pm 8.3$ & $43.3 \pm 8.5^{*}$ & $39.6 \pm 9.3$ & $3.8 \pm 2.3^{\S}$ & $26.4 \pm 10.4^{\S}$ \\
\hline Ob.S/BS (\%) & $11.0 \pm 3.9$ & $24.0 \pm 4.6$ & $3.8 \pm 2.6$ & $22.5 \pm 7.5$ & $35.4 \pm 7.8^{*}$ & $32.0 \pm 7.4$ & $2.2 \pm 1.5^{\S}$ & $20.5 \pm 9.3^{\S}$ \\
\hline MS/BS (\%) & $19.0 \pm 3.3$ & $31.0 \pm 6.6$ & $5.1 \pm 2.4$ & $31.1 \pm 6.7$ & $41.5 \pm 7.9^{*}$ & $39.4 \pm 9.0$ & $4.7 \pm 3.0^{\S}$ & $24.8 \pm 9.2^{\S}$ \\
\hline $\mathrm{BFR} / \mathrm{BS}\left(\mathrm{mm}^{3} / \mathrm{mm}^{2} /\right.$ year$)$ & $0.13 \pm 0.02$ & $0.24 \pm 0.07$ & $0.03 \pm 0.03$ & $0.24 \pm 0.05$ & $0.34 \pm 0.08^{*}$ & $0.28 \pm 0.08$ & $0.03 \pm 0.02^{\S}$ & $0.19 \pm 0.07^{\S}$ \\
\hline $\operatorname{MAR}(\mu \mathrm{m} /$ day $)$ & $1.9 \pm 0.1$ & $2.1 \pm 0.2$ & $1.7 \pm 0.3$ & $2.1 \pm 0.2$ & $2.2 \pm 0.2$ & $1.9 \pm 0.2^{\S}$ & $1.6 \pm 0.3^{\S}$ & $2.1 \pm 0.2$ \\
\hline Ac.f (N/year) & $6.5 \pm 1.0$ & $11.9 \pm 3.7$ & $1.5 \pm 0.9$ & $11.5 \pm 2.6$ & $15.4 \pm 3.8^{*}$ & $12.7 \pm 3.5$ & $1.3 \pm 1.0^{\S}$ & $9.5 \pm 3.6^{\S}$ \\
\hline ES/BS (\%) & $26.7 \pm 5.8$ & $42.2 \pm 4.7$ & $28.8 \pm 12.6$ & $38.0 \pm 4.5$ & $20.8 \pm 7.0^{*}$ & $19.8 \pm 6.7$ & $18.6 \pm 2.3$ & $34.6 \pm 4.0^{\S}$ \\
\hline N.Oc/BS (N/mm) & $2.8 \pm 1.1$ & $4.0 \pm 0.6$ & $2.7 \pm 1.1$ & $3.1 \pm 0.8$ & $2.2 \pm 0.7^{*}$ & $2.1 \pm 0.6$ & $2.2 \pm 0.4$ & $3.1 \pm 0.5^{\S}$ \\
\hline
\end{tabular}

Data are presented as mean $\pm \mathrm{SD}(N=7$ in pre of Sham, $N=8$ in pre of OVX and Wk 4 of Sham, and $N=10$ in other groups)

$B V / T V$ bone volume/tissue volume, $T b . T h$ trabecular thickness, $T b . S p$ trabecular separation, $B S / B V$ bone surface/bone volume, $O S / B S$ osteoid surface/bone surface, $O b . s / B S$ osteoblast surface/bone surface, $M S / B S$ mineralizing surface/bone surface, $B F R / B S$ bone formation rate/bone surface, $M A R$ mineral apposition rate, $A c$. $f$ activation frequency, $E S / B S$ eroded surface/bone surface and $N . O c / B S$ osteoclast number/bone surface

*Significant difference versus vehicle-treated OVX at Week 4 (unpaired t-test, $p<0.05$ )

${ }^{\S}$ Significant difference vs. PTH group at Wk 4 (Dunnett's multiple comparison test, $p<0.05$ ) 

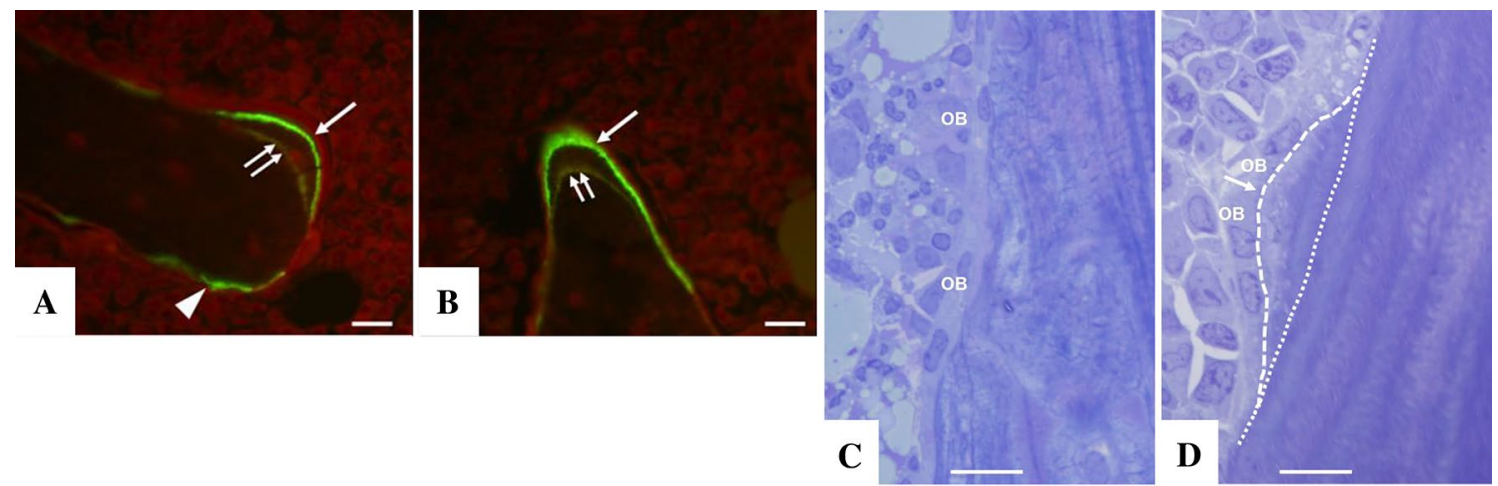

Fig. 5 Fluorescence microscopic observations in PTH-ELD group (a, b), on proximal tibias at Wk 8. Fluorescence by tetracycline label (a, b, double arrow) was observed on smooth arrest line and fluorescence by calcein label (a, b, single arrow) was observed on tetracycline label. This bone resorption independent bone formation is "minimodeling" and distinguished from "remodeling" which occurs on scal- loped cement line (a, arrow head). Scale bars show $20 \mu \mathrm{m}$ in $\mathbf{a}, \mathbf{b}$ and $10 \mu \mathrm{m}$ in c, d. Toluidine blue staining in PTH-PTH group (c) and PTH-ELD group (d) on proximal tibias at Wk 8. Local-convex bone formation encompassed by dotted lines (d), i.e., minimodeling, was observed in PTH-ELD. $O B$ osteoblast
A Ultimate Load (N)

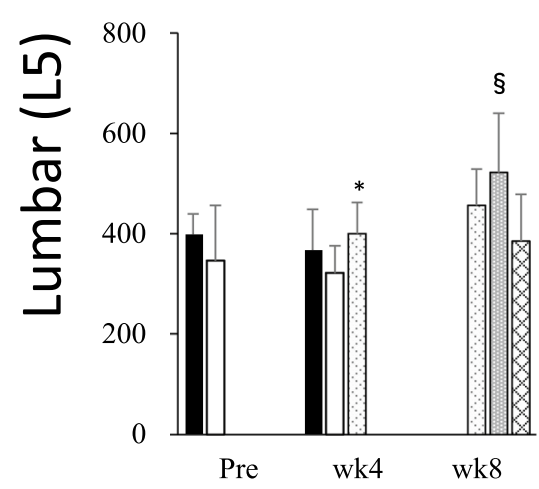

D Ultimate Load (N)

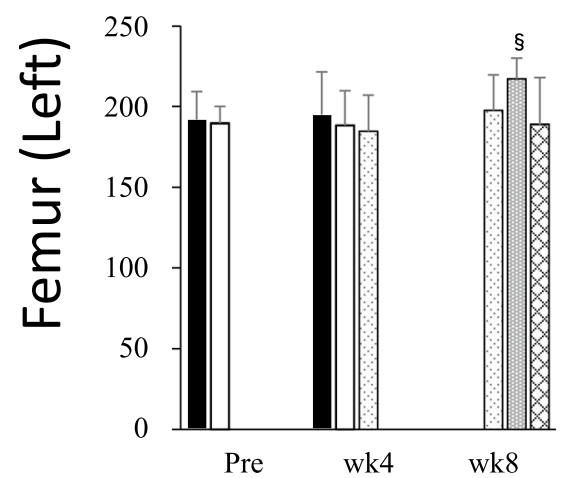

B Stiffness $(\mathrm{N} / \mathrm{mm})$

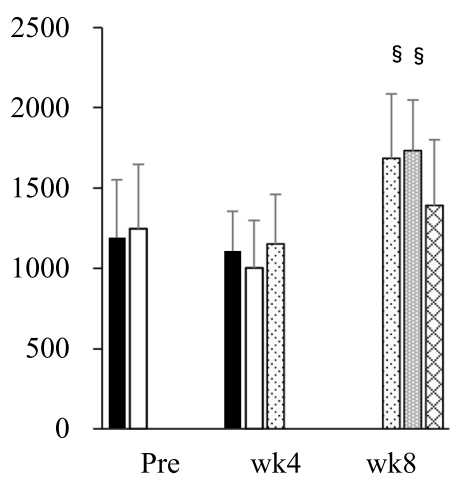

E Stiffness (N/mm)
C Energy (mJ)

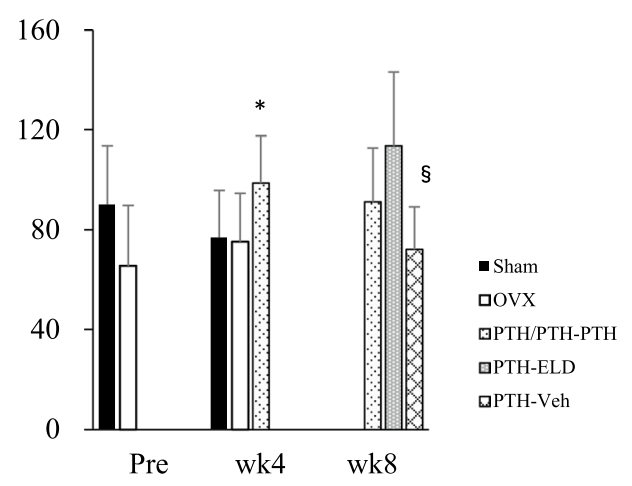

F Energy (mJ)
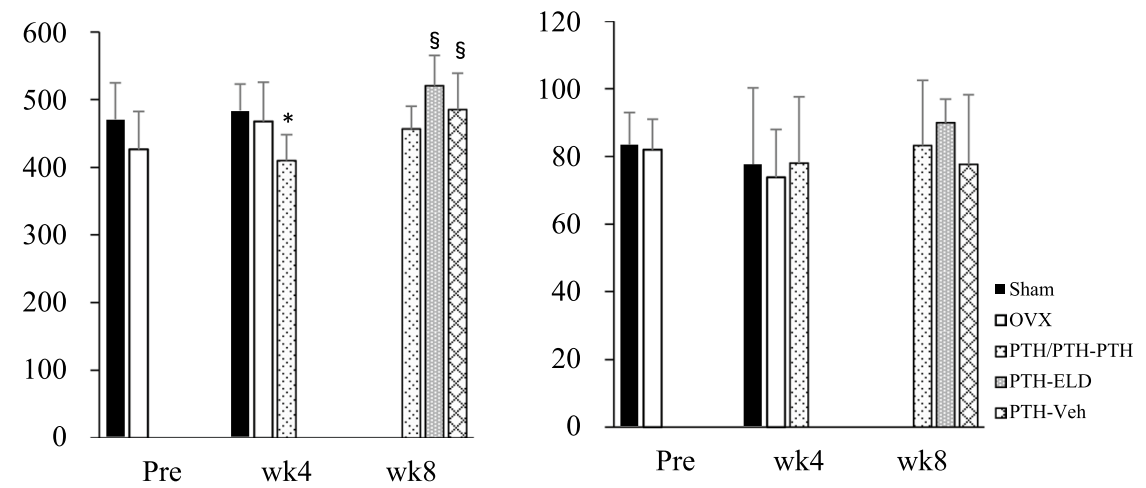

Fig. 6 Bone strength was assessed by compression test for the L5 lumbar vertebra and 3-point bending test for the middle femur. Data are presented as mean $+\mathrm{SD}(N=7$ in pre of Sham, $N=8$ in pre of OVX and Wk 4 of Sham, and $N=10$ in other groups). *Signifi- cant difference versus vehicle-treated OVX at Wk 4 (unpaired $t$-test, $p<0.05$ ). ${ }^{\S}$ Significant difference versus PTH group at Wk 4 (Dunnett's multiple comparison test, $p<0.05$ ) 
However, because of the limitations on the length of PTH treatment, alternative treatments have to be considered. In this study, we examined the effects of treatment with ELD following sequentially after PTH treatment in rats with osteoporosis induced by ovariectomy.

In these OVX rats, both osteoclastic bone resorption and reactive osteoblastic bone formation were increased, with the net result of this high bone turnover being a reduction in BMD. Treatment with PTH for 4 weeks increased the BMD of the lumbar spine and femur with an increase in bone formation and a reduction in bone resorption. However, this effect was not maintained after cessation of PTH treatment, and bone resorption especially was greatly increased. Switching to ELD after cessation of PTH maintained the BMD that had been increased by the PTH treatment in OVX rats. ELD reduced both bone resorption and bone formation parameters, implying that ELD induced lower bone turnover, and prevented bone loss mainly by reduction of bone resorption.

We analyzed the structural changes in the bones of OVX rats and the effects of anti-osteoporosis treatments with PTH and ELD and also the effects of PTH withdrawal. HE staining of the tibia revealed a reduction of trabecula bone in OVX rats. Treatment with PTH for 4 weeks seemed to increase the trabecular bone number and thickness. Although these effects of PTH were not maintained following the withdrawal of PTH treatment, switching to ELD was able to maintain the favorable effects of PTH. These results were quantitatively confirmed by microCT analysis in the femur.

It is known that PTH increases osteoblast numbers by inducing osteoblastogenesis, reducing osteoblast apoptosis, and reactivating quiescent lining osteoblasts $[2,26]$. The bone anabolic effects of PTH were confirmed by the increase in bone formation markers (Ob/BS, MS/BS, BFR/BS, Ac.f) in the bone histomorphometry of the proximal tibia. However anabolic effects of PTH in this study might be lower than other reports, generally PTH was reported to increase the bone mass parameters (e.g., BV/TV) until Sham level in OVX animals. Although the dosage of PTH in this study was lower than that of other groups [27, 28], we select this dose as a suitable dose to evaluate the bone volume or strength in OVX rats. Indeed, our previous studies using same PTH doses with this study, BV/TV in PTH group were almost same as Sham group [13, 29]. The difference of age of rats or experimental condition might influence to the anabolic effects of PTH. It is also reported that PTH has the potential to increase osteoclast numbers and bone resorption via the RANKL pathway [30]. However, in this study, PTH treatment reduced bone resorption, as demonstrated by the reduction of bone resorption parameters ES/BS and N.Oc/BS. Although PTH treatment did not enhance bone resorption, withdrawal of PTH enhanced the bone resorption parameters and increased numbers of TRAP-positive cells which were considered to be osteoclasts. There are conflicting reports about the influence that PTH has on bone resorption. Samadfam et al. reported that daily injection of PTH at a dose of $80 \mu \mathrm{g} / \mathrm{kg}$ increased both bone formation and bone resorption in OVX mice [27]. According to Iwaniec et al., administration of full-length PTH (75 $\mu \mathrm{g} / \mathrm{kg}$, three times perweek) for 12 weeks to OVX rats increased bone formation without upregulation of bone resorption in tibial cancellous bone [28]. Yamamoto et al. reported that dose and frequency of PTH administration affect the effect of PTH on bone metabolism in young adult normal mice [14]. In our study, PTH (human PTH1-34) was given to OVX rats at a dose of $10 \mu \mathrm{g} /$ $\mathrm{kg}$, five times per week. Thus, our results showing increased bone formation without a significant increase in bone resorption may have been affected by these conditions of dose, frequency, and animal.

Vitamin D plays important roles in calcium absorption from the intestine and in bone metabolism. Therefore, vitamin D analogs such as ELD or alfacalcidol ( $\alpha$-hydroxy vita$\min D_{3}$ ) are frequently used as osteoporosis treatments. It is reported that ELD suppresses bone resorption to a greater extent than alfacalcidol but with a similar effect on bone formation and calcium metabolism, resulting in a greater increase in BMD in OVX rats [31]. Moreover, the fracture incidence in ELD-treated osteoporosis patients was lower than that of alfacalcidol-treated patients in a 3-year doubleblind clinical trial [32].

In histomorphometry analysis, some of bone formation parameters (such as Ob,S/BS, BFR/BS and Ac.f) in Wk 4 of Sham was lower than those in pre of Sham. Although bone formation was gradually reduced according to the aging, changes of those parameters in this study seem too large. Sham animals were treated only citrate-phosphate buffer as vehicle of PTH and this vehicle is quite unlikely to influence for those parameters. The exact reason to explain this phenomenon is unclear. In this study, withdrawal of PTH treatment enhanced bone resorption and ELD prevented it. In our microCT data, PTH-PTH group showed higher BMD than that of 4 week PTH. Meanwhile, PTH-Veh group showed lower BMD than 4 week PTH (Fig. 4). According to the results from microCT and histomorphometry, PTH-PTH tends the superior of bone formation and $\mathrm{PTH}-\mathrm{Veh}$ group tends the superior of bone resorption. Concerning to PTH-ELD group, its BMD in microCT was not differed from 4wk PTH. Bone metabolic parameters in histomorphometry are almost same as 4wk Sham. It means that ELD reduced the increased bone-turnover by PTH and kept the normal level of bone-turnover. As the result of that ELD prevent the BMD reduction like $\mathrm{PTH}-\mathrm{Veh}$.

We performed the immunostaining of ALP to determine the osteoblast. In trabecular bone surface in PTH-PTH, two layers of ALP-positive cells were observed. Generally, osteoblast made monolayer, therefore some of 
ALP-positive cells in PTH-PTH group might be preosteoblast. Concerning to the histomorphometry, osteoblast must be reduced in ELD treatment; however, ALP-positive cells were observed in PTH-ELD. It means that ALP-positive cells in PTH-ELD might be other cells such as adipocyte rather than osteoblast. If that is case, ALP-positive cells should increase even in $\mathrm{PTH}-\mathrm{Veh}$ group because the increase of adipocyte in OVX rat bone is well known phenomenon. Mesenchymal stem cell can differentiate into both of osteoblast and adipocyte, and inverse relationship exists between the differentiation into osteoblast and adipocyte. Some researchers focused on the influence of vitamin D to the adipocyte and reported controversial results. For example, Ji et al. reported that vitamin D prevents the differentiation to adipocyte via reduction of PPAR $\gamma$ expression in 3T3-L1 preadipocyte [33]. Meanwhile, Narvaez et al. reported that vitamin D stimulates adipogenesis with induction of STEAP4 in human adipose derived mesenchymal progenitor cells [34]. The influence of vitamin $\mathrm{D}$ to the adipogenesis seems to differ by the experiment conditions such as cell type, concentration of vitamin D, experiment period or culture media components. Further research are required to identify the cell type of ALP-positive cells or to clarify the influence of adipocyte metabolisms on the bone protective effects of ELD.

Interestingly, bone strength parameters in the group switched to ELD tended to be higher than in the group that received no further treatment after cessation of PTH treatment and even in the group treated with PTH for 8 weeks. The gain in bone mechanical properties in the group switched to ELD seems to be comparatively greater than the gain in BMD. This means that it might not only be the increase in BMD but also the improvement in bone quality that contributed to the increase in bone strength in the ELD-treated group. The mechanisms through which ELD improves bone quality are not yet clear but enhancement of minimodeling might contribute to improving bone quality. Minimodeling is bone formation on quiescent bone surfaces that occurs independently of bone resorption $[16,17]$, and it is speculated to be one of the adaptive mechanisms through which bone strength is enhanced in response to mechanical stress [35]. ELD induces minimodeling in OVX monkeys, and this phenomenon is suggested to play some role in trabecular bone mineralization [18]. Increased minimodeling elicited by ELD is reported even in postmenopausal osteoporosis patients [36].

In conclusion, treatment with ELD sequentially following PTH withdrawal was able to maintain the BMD and bone mechanical properties in OVX rats.

Acknowledgements We thank Dr. H. Tomizawa-Shinohara, Dr. K. Serizawa, and Mr. M. Tashiro for their technical support.
Author Contributions SS, SH, and NA planned the study. SS, HH, TY, TH, ST, KE, and KY performed and analyzed the experiments. SS and KY wrote the first draft. All authors reviewed the results and approved the final version of the manuscript.

Funding This study was funded by Chugai Pharmaceutical Co., Ltd.

\section{Compliance with Ethical Standards}

Conflict of interest Norio Amizuka has received research grants from Chugai Pharmaceutical Co., Ltd. Sadaoki Sakai, Satoshi Takeda, Hitoshi Saito, Koichi Endo, Kenji Yogo are employees of Chugai Pharmaceutical Co., Ltd. Hiromi Hongo, Tomomaya Yamamoto, and Tomoka Hasegawa have no conflict of interest.

Human and Animal Rights and Informed Consent All animal experiments were performed in the laboratories of Chugai Pharmaceutical Co., Ltd. The experimental protocol was approved by the Institutional Animal Care and Use Committee at Chugai Pharmaceutical Co., which is accredited by the Association for Assessment and Accreditation of Laboratory Animal Care (AAALAC).

Open Access This article is distributed under the terms of the Creative Commons Attribution 4.0 International License (http://creativeco mmons.org/licenses/by/4.0/), which permits unrestricted use, distribution, and reproduction in any medium, provided you give appropriate credit to the original author(s) and the source, provide a link to the Creative Commons license, and indicate if changes were made.

\section{References}

1. Andreopoulou P, Bockman RS (2015) Management of postmenopausal osteoporosis. Annu Rev Med 66:329-342

2. Jilka RL (2007) Molecular and cellular mechanisms of the anabolic effect of intermittent PTH. Bone 40:1434-1446

3. Neer RM, Arnaud CD, Zanchetta JR, Prince R, Gaich GA, Reginster JY, Hodsman AB, Eriksen EF, Ish-Shalom S, Genant HK, Wang O, Mitlak BH (2001) Effect of parathyroid hormone (1-34) on fractures and bone mineral density in postmenopausal women with osteoporosis. N Engl J Med 344:1434-1441

4. Kaufman JM, Orwoll E, Goemaere S, San Martin J, Hossain A, Dalsky GP, Lindsay R, Mitlak BH (2005) Teriparatide effects on vertebral fractures and bone mineral density in men with osteoporosis: treatment and discontinuation of therapy. Osteoporos Int 16:510-516

5. Tashjian AH Jr, Goltzman D (2008) On the interpretation of rat carcinogenicity studies for human PTH(1-34) and human PTH(184). J Bone Miner Res 23:803-811

6. Finkelstein JS, Wyland JJ, Leder BZ, Burnett-Bowie SM, Lee H, Jüppner H, Neer RM (2009) Effects of teriparatide retreatment in osteoporotic men and women. J Clin Endocrinol Metab 94:2495-2501

7. Black DM, Bilezikian JP, Ensrud KE, Greenspan SL, Palermo L, Hue T, Lang TF, McGowan JA, Rosen CJ, PaTH Study Investigators (2005) One year of alendronate after one year of parathyroid hormone (1-84) for osteoporosis. N Engl J Med 353:555-565

8. Kurland ES, Heller SL, Diamond B, McMahon DJ, Cosman F, Bilezikian JP (2004) The importance of bisphosphonate therapy in maintaining bone mass in men after therapy with teriparatide [human parathyroid hormone(1-34)]. Osteoporos Int 15:992-997 
9. Adami S, San Martin J, Muñoz-Torres M, Econs MJ, Xie L, Dalsky GP, McClung M, Felsenberg D, Brown JP, Brandi ML, Sipos A (2008) Effect of raloxifene after recombinant teriparatide [hPTH(1-34)] treatment in postmenopausal women with osteoporosis. Osteoporos Int 19:87-94

10. Rittmaster RS, Bolognese M, Ettinger MP, Hanley DA, Hodsman AB, Kendler DL, Rosen CJ (2000) Enhancement of bone mass in osteoporotic women with parathyroid hormone followed by alendronate. J Clin Endocrinol Metab 85:2129-2134

11. Amugongo SK, Yao W, Jia J, Dai W, Lay YA, Jiang L, Harvey D, Zimmermann EA, Schaible E, Dave N, Ritchie RO, Kimmel DB, Lane NE (2014) Effect of sequential treatments with alendronate, parathyroid hormone (1-34) and raloxifene on cortical bone mass and strength in ovariectomized rats. Bone 67:257-268

12. Takano Y, Tanizawa T, Mashiba T, Endo N, Nishida S, Takahashi HE (1996) Maintaining bone mass by bisphosphonate incadronate disodium (YM175) sequential treatment after discontinuation of intermittent human parathyroid hormone (1-34) administration in ovariectomized rats. J Bone Miner Res 11:169-177

13. Takeda S, Sakai S, Tanaka K, Tomizawa H, Serizawa K, Yogo K, Urayama K, Hashimoto J, Endo K, Matsumoto Y (2017) Intermittent ibandronate maintains bone mass, bone structure, and biomechanical strength of trabecular and cortical bone after discontinuation of parathyroid hormone treatment in ovariectomized rats. Calcif Tissue Int 101:65-74

14. Yamamoto T, Hasegawa T, Sasaki M, Hongo H, Tsuboi K, Shimizu T, Ota M, Haraguchi M, Takahata M, Oda K, Luiz de Freitas PH, Takakura A, Takao-Kawabata R, Isogai Y, Amizuka N (2016) Frequency of teriparatide administration affects the histological pattern of bone formation in young adult male mice. Endocrinology 157:2604-2620

15. Ono Y (2014) Multifunctional and potent roles of the 3-hydroxypropoxy group provide eldecalcitol's benefit in osteoporosis treatment. J Steroid Biochem Mol Biol 139:88-97

16. de Freitas PH, Hasegawa T, Takeda S, Sasaki M, Tabata C, Oda K, Li M, Saito H, Amizuka N (2011) Eldecalcitol, a second-generation vitamin $\mathrm{D}$ analog, drives bone minimodeling and reduces osteoclastic number in trabecular bone of ovariectomized rats. Bone 49:335-342

17. Saito H, Takeda S, Amizuka N (2013) Eldecalcitol and calcitriol stimulates 'bone minimodeling,' focal bone formation without prior bone resorption, in rat trabecular bone. J Steroid Biochem Mol Biol 136:178-182

18. Saito M, Grynpas MD, Burr DB, Allen MR, Smith SY, Doyle N, Amizuka N, Hasegawa T, Kida Y, Marumo K, Saito H (2015) Treatment with eldecalcitol positively affects mineralization, microdamage, and collagen crosslinks in primate bone. Bone 73:8-15

19. Takeda S, Saito M, Sakai S, Yogo K, Marumo K, Endo K (2017) Eldecalcitol, an active vitamin D3 derivative, prevents trabecular bone loss and bone fragility in type I diabetic model rats. Calcif Tissue Int 101:433-444

20. Sakai S, Endo K, Takeda S, Mihara M, Shiraishi A (2012) Combination therapy with eldecalcitol and alendronate has therapeutic advantages over monotherapy by improving bone strength. Bone 50:1054-1063

21. Villanueva AR (1974) A bone stain for osteoid seams in fresh, unembedded, mineralized bone. Stain Technol 49:1-8

22. Parfitt AM, Drezner MK, Glorieux FH, Kanis JA, Malluche H, Meunier PJ, Ott SM, Recker RR (1987) Bone histomorphometry: standardization of nomenclature, symbols, and units. Report of the ASBMR Histomorphometry Nomenclature Committee. J Bone Miner Res 2:595-610
23. Amizuka N, Kwan MY, Goltzman D, Ozawa H, White JH (1999) Vitamin D3 differentially regulates parathyroid hormone/parathyroid hormone-related peptide receptor expression in bone and cartilage. J Clin Invest 103:373-381

24. Oda K, Amaya Y, Fukushi-Irie M, Kinameri Y, Ohsuye K, Kubota I, Fujimura S, Kobayashi J (1999) A general method for rapid purification of soluble versions of glycosylphosphatidylinositol-anchored proteins expressed in insect cells: an application for human tissue-nonspecific alkaline phosphatase. J Biochem 126:694-699

25. Amizuka N, Takahashi N, Udagawa N, Suda T, Ozawa H (1997) An ultrastructural study of cell-cell contact between mouse spleen cells and calvaria-derived osteoblastic cells in a co-culture system for osteoclast formation. Acta Histochem Cytochem 30:351-362

26. Pettway GJ, Schneider A, Koh AJ, Widjaja E, Morris MD, Meganck JA, Goldstein SA, McCauley LK (2005) Anabolic actions of PTH (1-34): use of a novel tissue engineering model to investigate temporal effects on bone. Bone 36:959-970

27. Samadfam R, Xia Q, Goltzman D (2007) Co-treatment of PTH with osteoprotegerin or alendronate increases its anabolic effect on the skeleton of oophorectomized mice. J Bone Miner Res 22:55-63

28. Iwaniec UT, Samnegård E, Cullen DM, Kimmel DB (2001) Maintenance of cancellous bone in ovariectomized, human parathyroid hormone $[\mathrm{hPTH}(1-84)]$-treated rats by estrogen, risedronate, or reduced $\mathrm{hPTH}$. Bone 29:352-360

29. Hasegawa T, Yamamoto T, Sakai S, Miyamoto Y, Hongo H, Qiu Z, Abe M, Takeda S, Oda K, de Freitas PHL, Li M, Endo K, Amizuka N (2018) Histological effects of the combined administration of eldecalcitol and a parathyroid hormone in the metaphyseal trabeculae of ovariectomized rats. J Histochem Cytochem. https ://doi.org/10.1369/0022155418806865

30. O'Brien CA, Nakashima T, Takayanagi H (2013) Osteocyte control of osteoclastogenesis. Bone 54:258-263

31. Uchiyama Y, Higuchi Y, Takeda S, Masaki T, Shira-Ishi A, Sato K, Kubodera N, Ikeda K, Ogata E (2002) ED-71, a vitamin D analog, is a more potent inhibitor of bone resorption than alfacalcidol in an estrogen-deficient rat model of osteoporosis. Bone 30:582-588

32. Matsumoto T, Ito M, Hayashi Y, Hirota T, Tanigawara Y, Sone T, Fukunaga M, Shiraki M, Nakamura T (2011) A new active vitamin D3 analog, eldecalcitol, prevents the risk of osteoporotic fractures - a randomized, active comparator, double-blind study. Bone 49:605-612

33. Ji S, Doumit ME, Hill RA (2015) Regulation of adipogenesis and key adipogenic geneexpression by 1,25 -dihydroxyvitamin $\mathrm{D}$ in 3T3-L1 cells. PLoS ONE 10:e126142

34. Narvaez CJ, Simmons KM, Brunton J, Salinero A, Chittur SV, Welsh JE (2013) Induction of STEAP4 correlates with 1,25-dihydroxyvitamin D3 stimulation of adipogenesis in mesenchymal progenitor cells derived from human adipose tissue. J Cell Physiol 228:2024-2036

35. Frost HM (1990) Skeletal structural adaptations to mechanical usage (SATMU): 1. Redefining Wolff's law: the bone modeling problem. Anat Rec 226:403-413

36. Hikata T, Hasegawa T, Horiuchi K, Fujita N, Iwanami A, Watanabe K, Ishii K, Nakamura M, Amizuka N, Matsumoto M (2016) Histomorphometric analysis of minimodeling in the vertebrae in postmenopausal patients treated with anti-osteoporotic agents. Bone Rep 5:286-291 